\title{
Discussion on Relativity of Time Interval in the Theory of Relativity
}

\author{
Zhonggang $\mathrm{Li}^{1}$ \\ ${ }^{1}$ Technology Department, Silkworm Research Institute, Beiheishan Village, Dasheng Town, Anqiu City, \\ Shandong Province, 262128, China \\ Correspondence: Zhonggang Li, Technology Department, Silkworm Research Institute, Beiheishan Village, \\ Dasheng Town, Anqiu City, Shandong Province, 262128, China. E-mail: 13465701349@163.com
}

Received: September 9, 2019

Accepted: September 26, 2019 Online Published: September 30, 2019

doi:10.5539/apr.v11n5p41

URL: http://dx.doi.org/10.5539/apr.v11n5p41

\begin{abstract}
Einstein's principle of relativity is an important fundamental of relativity. It can also be said that relativity is based on the assumption of the principle of relativity. This assumption can be represented by a diagram of "relativity of time interval", as shown in Fig. $a$. According to the theory of relativity, a flash emits from the light source in the moving car and returns to the light source after being reflected by the mirror on the roof. The person in the train thinks that the flash returns to the light source along a vertical line, and the person off the train thinks that the flash returns to the light source along the $A M B$ curve. The time taken in the two routes is inconsistent. In fact, it is a preconception. Einstein believes that the flash will undoubtedly return to the light source, which is a matter of course and need not be discussed. Therefore, he did not think more and immediately carried out the next research on the time taken inside and outside the train. Here's where the problem arose. Is there an experimental evidence? If the flash does return to the light source, then the next analysis carried out by Einstein is correct and impeccable. However, if the flash does not return to the light source, then Einstein's next analysis and the whole theory of relativity make no sense.
\end{abstract}

Keywords: velocity, time, the theory of relativity, velocity of light, reference system

Einstein's principle of relativity is an important fundamental of relativity. It can also be said that relativity is based on the assumption of the principle of relativity. This assumption can be represented by a diagram of "relativity of time interval", as shown in Fig. a. According to the theory of relativity, a flash emits from the light source in the moving car and returns to the light source after being reflected by the mirror on the roof. The person in the train thinks that the flash returns to the light source along a vertical line, and the person off the train thinks that the flash returns to the light source along the $A M B$ curve. The time taken in the two routes is inconsistent. In fact, it is a preconception. Einstein believes that the flash will undoubtedly return to the light source, which is a matter of course and need not be discussed. Therefore, he did not think more and immediately carried out the next research on the time taken inside and outside the train. Here's where the problem arose. Why did Einstein firmly believe that the flash in a moving car will return to the light source? Is there an experimental evidence? If the flash does return to the light source, then the next analysis carried out by Einstein is correct and impeccable. However, if the flash does not return to the light source, then Einstein's next analysis and the whole theory of relativity make no sense. Therefore, it is of primary importance to verify whether the flash in the moving car returns to the light source. The photon in a high-speed state is essentially different from the ball in low-speed state. We shall not take for granted that the photon must return to the starting point like a ball. Therefore, it is necessary to carry out experiments for verification. Actually, there may be two conclusions in the diagram of "relativity of time interval" of relativity theory: The flash may or may not return to the light source. It can be said that the whole destiny of relativity is closely related to this diagram. This experiment is very necessary but has never been verified and has been ignored for a long time. Einstein identified the first possibility groundlessly when it was up in the air and arbitrarily abandoned the second possibility. It is the first serious negligence by Einstein. Then, Einstein derived the formula 
$\Delta t=\frac{\Delta t^{\prime}}{\sqrt{(v)^{2}}}$, which can be interpreted in two ways: The first interpretation is when $1-\left(\frac{v}{c}\right)^{2}<1$, then $\Delta t>$ $\sqrt{1-\left(\frac{v}{c}\right)}$

$\Delta t^{\prime}$. The other interpretation is that only when $v=0$, the formula is true, i.e. $\Delta t=\Delta t^{\prime}$. That is, only when the car is stationary, the flash will return to the light source. Einstein was more than twenty with rich imagination then but he would rather believe $\Delta t>\Delta t^{\prime}$ than $\Delta t=\Delta t^{\prime}$, identifying the first interpretation out of thin air again and abandoning the second interpretation, so he lost the last opportunity of correcting his theory. After that, Einstein went further on the foundation with continuous uncertainties and established his theory of relativity, which has been around for a century.

The exact analogy of the reflection of a photon moving at high velocity to the rebound of a ball at low velocity by extrapolating the Galilean principle of relativity summarized in low-speed state is not reliable and should be taken seriously. The law of Ockham's Razor (i.e. the "law of thrift" commonly applied in science) shows that the most possible, simplest and most frugal interpretation is the best explanation, unless there is evidence that this simplest explanation is wrong. Einstein should have chosen the case when $v=0, \Delta t=\Delta t^{\prime}$. However, Einstein chose another explanation. To be honest, it is not scientific nor logical. Consequently, it is not surprising that many illogical conclusions have been derived from the theory of relativity, because the fundamentals of relativity are something unreasonable. Unfortunately, some people prefer to believe some illogical theories. Maybe they like to hold different views against logic and reality in their nature.

\section{The diagram of relativity of time interval in the theory of relativity is shown in Fig. (a).}
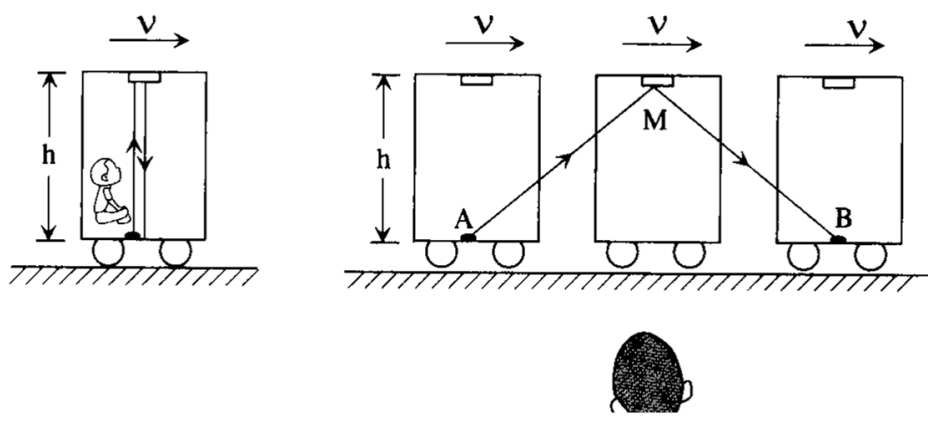

Fig. A The person in the train thinks that the flash reaches the mirror and is reflected both in the vertical direction

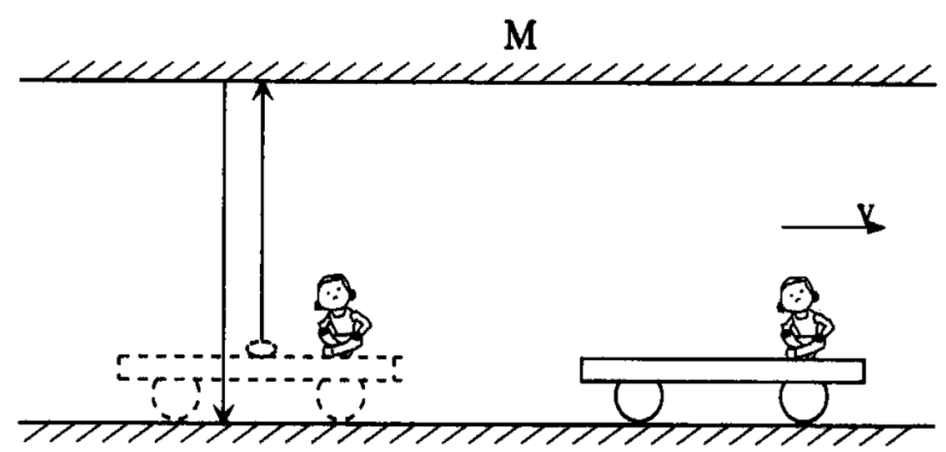

Fig. B The person off the train thinks that the light is transmitted in the slanting direction

The diagram of the theory of this text on the flash in a car is shown in Fig. (b). 
Photons are not affected by the inertial system of the car from the moment they leave the light source. When photons are emitted from the light source of the car, the car leaves. Photons travel in the given direction (vertically upwards) and then arrive at the original light source on the railway along the same path after being reflected. They do not move with the car and are not transmitted in the slanting direction, which is completely different from the motion of the ball with the train. The person in the train does feel that the photons have drifted backwards, but it is only his personal feeling which is a matter of consciousness. Consciousness has no effects on external material world. Although the car in this figure is not equipped with a roof, the effect produced and principle applied in it are the same as those in the car with a roof. Light velocity is a concern that spans the inertial system. It is not good to artificially define some inertial systems when studying light velocity and no preconceptions must be taken. It is even impossible to regard inertial system as an "independent kingdom". That is to say, the person off the train observes that the flash does not return to the light source and the flash in the moving car does not travel along the AMB curve. The relativity of time does not exist. The relativistic space-time view is only a pure mathematical model rather than an objective fact. Since the fundamentals of relativity do not exist, the relativity is also impractical. The theory of relativity is purely a successful mathematical model.

I want to analyze it theoretically to better understand the success and failure of relativity.

In fact, Einstein's principle of relativity is also contradictory to the principle of constancy of light velocity. The famous Michelson-Morley experiment and other experiments have demonstrated that the velocity of light is always the same regardless of the relative motion of the light source, that is, the motion of the light source does not affect the velocity of light. According to Einstein's principle of relativity, the direction of light changes due to the motion of the light source and the velocity of light also changes. As shown in Fig. C, a beam of light is emitted perpendicular to the direction of motion of a car moving linearly at a speed of $0.9 c$ per second and is reflected by $M$ on the top plate of the car, the reflected light must return to the light source in the car according to Einstein's principle of relativity. Then, if observed off the train and the car runs a distance of $0.9 c \times 2$ seconds within 2 seconds, the reflected light must travel a distance of about $1.3 c \times 2$ seconds in 2 seconds, which is obviously faster than light.

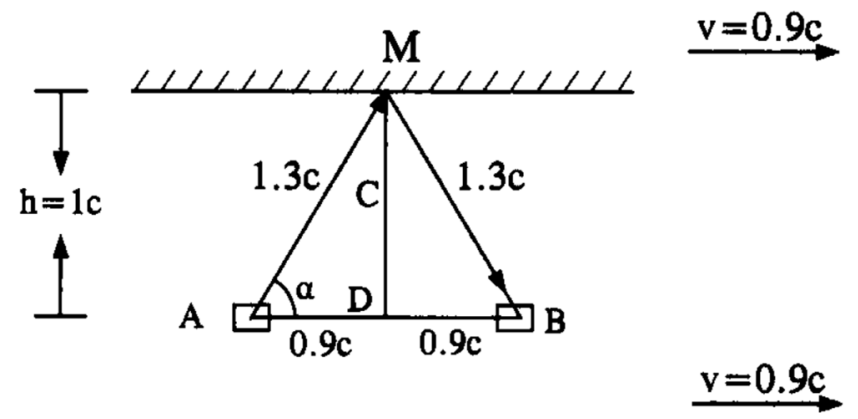

Fig. (c) A huge carriage is composed of a high-velocity car and a $M 300,000 \mathrm{~km}$ away.

This is an ideal experiment based on the theory of relativity.

When studying the relationship between $T$ and $T^{\prime}$, the Pythagorean theorem is used to calculate the relationship with the result of $T>T^{\prime}$, i.e. $A M>M D$. Because $A M=c T, M D=c T^{\prime}, T>T^{\prime}$. We see that Einstein compared the relationship between $A M$ observed off the car and $M D$ observed in the car, which essentially fixed the velocity of light $c$ first. The distance becomes larger and then the time $\mathrm{T}$ is increased. We should not follow his way of thinking. We study $A M$ and $A D$ both observed outside the train, so the time T corresponding to $A M$ and $A D$ is consistent. We fix the time $\mathrm{T}$ first. If the train runs a distance of $A M$ within the fixed time $\mathrm{T}$ which it can only complete at super velocity of light, a strange conclusion that the light is faster than the velocity of light will inevitably be drawn. That is to say, if we agrees that time is inconsistent according to the principle of relativity, we can also admit that the velocity of light is variable or super beyond light. It is obviously wrong and contradictory. Therefore, the postulates and diagrams of Einstein's principle of relativity are untenable, which would lead physics further astray.

Super velocity of light or super time is incredible, but the conclusion of super velocity of light obviously contradicts the principle of constancy of light velocity, which is inconsistent with the facts such as 
Michelson-Morley Experiment. The conclusion of super time is beneficial to the theory of relativity, so Einstein admitted the conclusion of super time observed outside the train.

We should pay attention to an interesting phenomenon here. If we fix the parameters observed in the train and then derive them to apply to the outside of the train, the conclusion of super time will inevitably be drawn, that is, the time observed outside the train gets longer, which is inconsistent with the time observed in the train. In addition, the derivation process is strict and impeccable, which is one of the reasons why the theory of relativity remains invincible for a hundred years. But if we fix the parameters observed outside the train and then derive them to apply to the inside of the train, we will inevitably reach the conclusion of super velocity of light and such derivation process is also invulnerable. Here I emphasize three points to avoid doubts by others: First, it is true that a car moving at a speed of $0.90 c$ runs a distance of $0.90 c \times 1$ second in one second and a distance of $0.90 c \times 2$ seconds in 2 seconds. Second, it is true that a beam of light is emitted from a car to the object $M \quad 300,000$ kilometers away and will reach $M$ after 1 second. In other words, it is right that a beam of light emitted from the earth will reach the moon after more than one second. Third, according to Einstein's principle of relativity, it is true that an isosceles triangle similar to that in Fig. $\mathrm{C}$ will be definitely formed if the flash returns to the light source in the car. Therefore, according to the Pythagorean theorem, $A M$ is necessarily greater than the distance of $0.90 c$ $\times 1$ second, i.e. $M D$, so the conclusion that the light is super beyond light velocity can be drawn. Let us think about them. The first point is indisputable. The second point is supported by Michelson-Morley Experiment, that is, the motion of light source does not affect the velocity of light. Then, why is the conclusion of super velocity of light contradicting facts derived? The third point must be problematic, that is, Einstein's principle of relativity is wrong. The flash will not return to the light source in the car.

Generally speaking, experiments are specially effective to test whether pure theories are correct or not. However, it is often difficult to test the correctness under the historical conditions of the pure theories. In another way, we can find flaws from a theoretical perspective, but should never be drawn by their ideas. If we follow their ideas, we will often find them impeccable. This is because we follow the rules and routes set by them. For example, if a cat catches a mouse according to the rules set by mice, can it catch the mouse? I'm afraid it is quite difficult. It can not catch the mouse and even worse it is controlled by the mouse from another point of view. For the theory of relativity, are we cats or mice? I know many friends who started as opponents of relativity and finally become staunch supporters of relativity. There are similar examples in our life. For example, if we are led in a long alley we are not familiar with, we will be dizzy after turns and have to believe and rely on the people who lead us.

In short, the theory of foron believes that Einstein's principle of relativity is idealized, that is to say, Galilean principle of relativity was summarized when an object is moving at low velocity, so Einstein reasoned it by analogy in a rash way; Einstein's extrapolation was to defend relativity of "simultaneity" and relativity of "time interval" proposed by him. He set up the framework of the principle of relativity and then defended it which could not be refuted. But facts speak louder than words. In fact, the velocity of light is a consideration that crosses inertial systems. Galilean principle of relativity summarized in low-velocity macroscopic state was arbitrarily analogized to that in high-velocity state as it was, which is wrong.

For example, there is a consideration in the relativity of simultaneity. According to Galilean principle of relativity, when two iron balls are simultaneously rolled at the same speed from the middle to both ends of the car moving at a uniform speed in a straight line, then a person in the car will see that the balls reach both ends of the car simultaneously.

What about the situation if observed on the ground?

Assuming that the train uniformly moves to the right in a straight line, the person on the ground observes that the velocity at which the iron ball moves to the right is $\left(V+V^{\prime}\right)$, where $V$ represents the velocity of the iron ball and $V^{\prime}$ represents the velocity of the train. The displacement of the iron ball is $\left(S_{1}+S_{2}\right)$, where $S_{1}$ is half the length of the car with a value of $V T, S_{2}$ is the distance the train moves in the time of $T$ with a value of $V^{\prime} T$; similarly, the velocity of the iron ball moving to the left is $\left(V-V^{\prime}\right)$ and the displacement is $\left(S_{1}-S_{2}\right)$. Thus,

The time taken by the iron ball to reach the right end is: $\frac{V T+V^{\prime} T}{V+V^{\prime}}=T$

The time taken by the iron ball to reach the left end is: $\frac{V T-V^{\prime} T}{V-V^{\prime}}=T$ 
The time taken by the iron ball to reach the left end is equal to that taken by the iron ball to reach the right end, so the time taken by the iron balls to reach both ends is the same when observed on the ground, that is, the iron balls reach both ends of the car simultaneously, and there is no relativity of "simultaneity" as shown in Fig. (d)

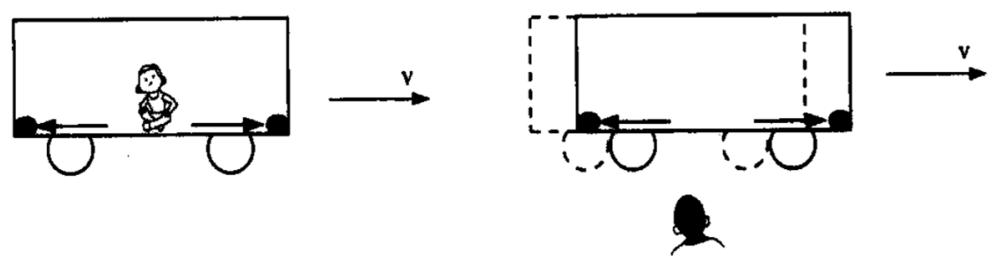

Fig. A The observer in the train sees that the iron balls reach the front and rear walls of the car at the same time.

Fig. B The observer off the train sees that the iron balls also reach the front and rear walls of the car at the same time.

Fig. (d)

The reason is because the balls are a part of this inertial system. Strictly speaking, the emitted light does not belong to this inertial system as the velocity of light is not affected by the velocity of the train, so the photons emitted from the light source in the middle of the car will never reach the two ends of the car simultaneously (even if observed in the train). The observations by persons inside and outside the train are still the same, that is, the relativity of "simultaneity" does not exist, which is consistent with the result of iron ball experiment. One event will never bring about two results at the same time. In case of contradictions between theory and facts, it should be said that the theory is wrong. Facts do not necessarily follow the theory with word game and sophistry.

Let us discuss the relativity of time interval. It is obvious when a rubber ball experiment is made in the car mentioned above. When a person exerts his strength to throw a rubber ball, the ball bounces to the roof of the car and rebounds to the original place. The travel distance of the ball observed on the ground is really longer than that observed in the car. However, the observer on the ground thinks that the motion of the rubber ball is resultant, so is its velocity which is greater than that observed in the car. Therefore, the time taken by the rubber ball traveling the long distance observed on the ground is the same as that taken by the rubber ball traveling the short distance observed in the car, so there is no relativity of time interval. The description of an ideal experiment by using light is still wrong according to relativity. In fact, the travel route taken by photons is different from that taken by the rubber ball. Although light always travels in a vertical line, the trajectory of change in the position a photon relative to the car is a slanting line rather than a vertical line. When observed in the car, the photon is to drift backwards. When observed outside the car, the photon is also to drift backwards relative to the car. The observations are consistent, that is, the time interval is the same. Light is not affected by the velocity of the train, just as photons do not belong to this inertial frame. That is to say, light is transmitted upwards in a vertical line and returns to the light source relative to the railway. The photons drift backwards in the car in a slanting line relative to the car. During the travel of the photons, the car has moved forward a certain distance as shown in Fig. b.

The rubber ball moves forward with the car as it is affected by the velocity of the car; the photons are not affected by the velocity of the car, so they do not travel with the car while they propagate and return in the original vertical line. From the angle of the moving car, the photons seem to drift backward. The theory of relativity believes that the motion of photons is basically the same as that of the rubber ball. It shows that Einstein regarded a photon as a rubber ball.

As shown in Fig. e, the person in the train thinks that the time taken by the rubber ball which reaches the roof of the car and rebounds to the original place is $\Delta t^{\prime}, \Delta t^{\prime}=\frac{2 h}{v^{\prime}}$ if the velocity of the rubber ball is $v^{\prime}$. 

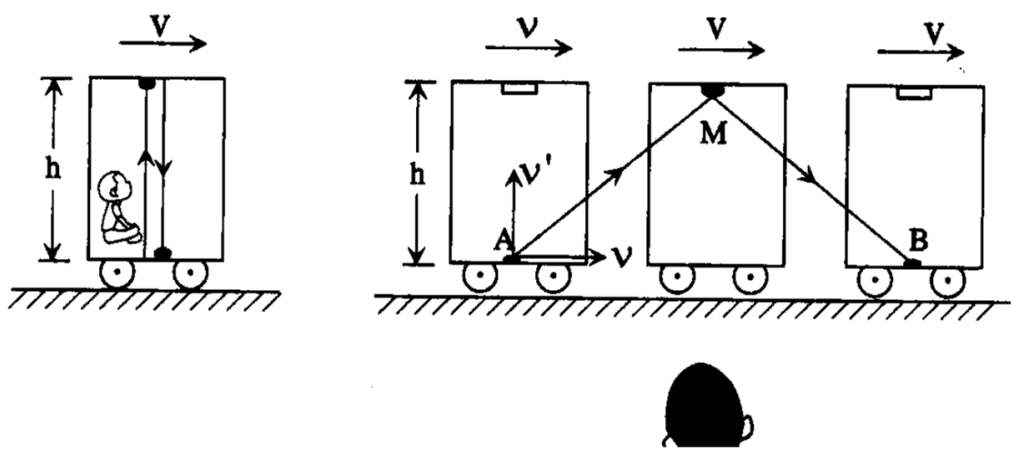

Fig. A The person in the train sees that the rubber ball reaches the roof of the car and rebounds to the original place both in the vertical direction.

Fig. B The person off the train sees that the rubber ball moves in the slanting direction.

Fig. (e)

For the observer on the ground, the rubber ball moves along the $A M B$. If the velocity $v^{\prime}$ of the rubber ball is equal to that $v$ of the train, the resultant velocity of the upward velocity $v^{\prime}$ and the train velocity $v$ is $V=\sqrt{v^{\prime 2}+v^{2}}=\sqrt{2} v^{\prime}$, and the distance of the rubber ball is $A M B=2 \times \sqrt{2} h$. Then the round-trip time of the rubber ball measured by the observer on the ground is

$$
\Delta t=\frac{2 \times \sqrt{2} h}{\sqrt{2} v^{\prime}}=\frac{2 h}{v^{\prime}}=\Delta t^{\prime}
$$

That is, with respect to the time taken by the rubber ball which bounces to the top and returns to the original place, the person on the ground and the other person in the train have consistent measurements, which have no relativity of time interval.

Here, the experiments of a rubber ball and an iron ball in the car prove that the "relativity of time" does not exist, so does the ideal experiment of light propagation in the car, which are contrary to Einstein's point of view. Some readers still have doubts about my viewpoint. In fact, your doubts are justified. The experiment of a rubber ball is really rough and the rules summarized at low velocity cannot be directly applied to the situation at high velocity. However, I want to know the reason why people believe Einstein's viewpoint of completely equating a high-velocity photon to a low-velocity rubber ball and directly applying the Galilean principle of relativity to the situation at high velocity. To say the least, Einstein used two standards or two logics to treat a photon as either a rubber ball or a photon whenever it was good for his description.

Taking the ideal experiment of "relativity of time interval" as an example, if observed outside the train, the direction of the flash actually results from the motion direction of the car and the upward direction of the flash. Here, it was still difficult for Einstein to abandon the classic physics theory about motion synthesis. He regarded a photon as a rubber ball indirectly recognizing that the flash direction would be affected by the light source of motion. Although a resultant direction is used to solve the direction problem, Einstein did not consider the velocity of the car in regard to velocity problem and believed that the velocity of light still remains the same. This explanation is based on two logics, which is nondescript or arbitrary. If we violate the "law of thrift" generally applied in science, choose the idea beneficial to our own theories in the absence of experiment evidence, arbitrarily abandon and neglect the idea unfavorable to our own theories and maintain the relativity based on the rash assumption of a beam of reflected light, we will finally get regrettable results. Although some calculation results are fairly consistent with the facts, it does not mean that the whole theory is correct, because there are many examples of satisfactory calculation results but wrong theories.

\section{Conclusions}

In short, when discussing the "relativity of simultaneity", the insistence that photons will reach both ends of the car at the same time is only a subjective judgment after optionally extrapolating Galilean principle of relativity. (If someone desperately identifies it even if he knows that it is not necessarily right, he may have an emotional or 
mental problem). In addition, when discussing the "relativity of time interval", it is not appropriate to identify the time of light propagation becomes longer if the travel trajectory of a photon becomes longer relative to the car. The seemingly more distance traveled due to the motion of the car does not affect the velocity of light and time. The velocity of light is constant. Outside events are the matters of their own and have no relation with light. It doesn't count that the light has traveled a long distance relative to the train. Otherwise the light is not transmitted in a straight line. For example, if we wave our hands to a certain object in the distance (In general, the wave indicates a motion along a curve.), then isn't the trajectory of the object relative to the hands a curve? But it has no effect on it. However, the related calculations of relativity are based on this assumed route of transmission in the slanting direction.

If you still hold on to the space-time view of relativity, I ask you another question: According to the space-time view of relativity, the time course in the train becomes slower and that on the ground is faster because the train is moving. However, the ground is moving by taking the train as the reference object, does the time course on the ground become slower and is the time course in the train faster? So which is faster or slower? What is the significance of such contradictory relativity of time? The word of "relativity" does not mean a privilege that is not logical or unreasonable. In order to straighten out a ridiculous theory, several absurd reasons have to be made up, which ultimately confuses ourselves leading to the loss of scientific spirit and deviation from basic facts. In fact, the theory of relativity has raised the inertial frame to an independent status.

\section{References}

Li, Z. G. Disagreements in the foundation of physics. America: International Institute for Science, Technology.

\section{Copyrights}

Copyright for this article is retained by the author(s), with first publication rights granted to the journal.

This is an open-access article distributed under the terms and conditions of the Creative Commons Attribution license (http://creativecommons.org/licenses/by/4.0/). 